\title{
Personalizing Evidence for Shoulder Fracture Patients Using an Extended Instrumental Variable Causal Forest Algorithm
}

\author{
John M. Brooks ${ }^{1}$, Cole G. Chapman² ${ }^{2}$ Sarah Floyd ${ }^{3}$, Brian K. Chen ${ }^{4}$, Charles A. Thigpen ${ }^{5}$, \\ and Michael Kissenberth ${ }^{6}$ \\ ${ }^{1}$ Center for Effectiveness Research in Orthopaedics - Arnold School of Public Health \\ Greenville, South Carolina United States \\ ${ }^{2}$ University of Iowa \\ ${ }^{3}$ Clemson University College of Behavioral Social and Health Sciences \\ ${ }^{4}$ University of South Carolina Arnold School of Public Health \\ ${ }^{5}$ University of South Carolina \\ ${ }^{6}$ Prisma Health
}

October 20, 2021

\begin{abstract}
Objective: To assess the ability of an extended Instrumental Variable Causal Forest Algorithm (IV-CFA) to provide personalized evidence of early surgery effects on benefits and detriments for elderly shoulder fracture patients. Data Sources/Study Setting: Population of 72,751 fee-for-service Medicare beneficiaries with proximal humerus fractures (PHFs) in 2011 who survived a 60-day treatment window after an index PHF and were continuously Medicare fee-for-service eligible over the period 12 months prior to index to the minimum of 12 months after index or death. Study Design: IV-CFA estimated early surgery effects on both beneficial and detrimental outcomes for each patient in the study population. Classification and regression trees (CART) were applied to these estimates to create patient reference classes. Two-stage least squares (2SLS) estimators were applied to patients in each reference class to scrutinize the estimates relative to the known 2SLS properties. Principal Findings: This approach uncovered distinct reference classes of elderly PHF patients with respect to early surgery effects on benefit and detriment. Older, frailer patients with more comorbidities, and lower utilizers of healthcare were less likely to gain benefit and more likely to have detriment from early surgery. Reference classes were characterized by the appropriateness of early surgery rates with respect to benefit and detriment. Conclusions: Extended IV-CFA provides an illuminating method to uncover reference classes of patients based on treatment effects using observational data with a strong instrumental variable. This study isolated reference classes of new PHF patients in which changes in early surgery rates would improve patient outcomes. The inability to measure fracture complexity in Medicare claims means providers will need to discuss the appropriateness of these estimates to patients within a reference class in context of this missing information.
\end{abstract}

\section{Hosted file}

paper_draft_v6.pdf available at https://authorea.com/users/435586/articles/538503personalizing-evidence-for-shoulder-fracture-patients-using-an-extended-instrumentalvariable-causal-forest-algorithm

\section{Hosted file}

A_Table_1_2_3_4_5_6.pdf available at https://authorea.com/users/435586/articles/538503personalizing-evidence-for-shoulder-fracture-patients-using-an-extended-instrumentalvariable-causal-forest-algorithm 